\title{
Weighted Cumulative Residual (Past) Inaccuracy For Minimum (Maximum) of Order Statistics
}

\author{
Safieh Daneshi ${ }^{1,2}$, Ahmad Nezakati ${ }^{1}$, Saeid Tahmasebi ${ }^{3, *}$ \\ ${ }^{1}$ Department of Statistics, Shahrood University of Technology, Shahrood, Iran \\ 2 Bushehr University of Medical Sciences, Bushehr, Iran \\ ${ }^{3}$ Department of Statistics, Persian Gulf University, Bushehr, Iran
}

\begin{abstract}
In this paper, we propose a measure of weighted cumulative residual inaccuracy between survival function of the first-order statistic and parent survival function $\bar{F}$. We also consider weighted cumulative inaccuracy measure between distribution of the last- order statistic and parent distribution $F$. For these concepts, we obtain some reliability properties and characterization results such as relationships with other functions, bounds, stochastic ordering and effect of linear transformation. Dynamic versions of these weighted measures are considered.
\end{abstract}

Keywords Cumulative inaccuracy, Order statistics, Empirical approach.

AMS 2010 subject classifications 62N05, 62B10, 94A17

DOI: $10.19139 /$ soic-2310-5070-695

\section{Introduction}

Let $X$ denote the lifetime of a device or a system with probability density function (pdf) $f$ and cumulative distribution function (cdf) $F$, respectively. Then, the differential entropy known as Shannon entropy, is defined by Shannon [18] as follows:

$$
H(X)=-\int_{0}^{+\infty} f(x) \log f(x) d x,
$$

where, by convention, $0 \log 0=0$. Di Crescenzo and Longobardi [6] introduced the concept of weighted differential entropy which is given by

$$
H^{w}(X)=-\int_{0}^{+\infty} x f(x) \log f(x) d x
$$

Recently, new measures of information are proposed in literatures: replacing the pdf by the survival function $\bar{F}=1-F$ in Shannon entropy, the cumulative residual entropy (CRE) is defined by Rao et al. [16] as

$$
\mathcal{E}(X)=\int_{0}^{+\infty} \bar{F}(x) \Lambda(x) d x,
$$

where $\Lambda(x)=-\log \bar{F}(x)$. Properties of the CRE can be found in [13], [15], and [22]. A new information measure similar to CRE has been proposed by Di Crescenzo and Longobardi [7] as follows:

$$
\mathcal{C E}(X)=\int_{0}^{+\infty} F(x) \tilde{\Lambda}(x) d x
$$

\footnotetext{
*Correspondence to: Saeid Tahmasebi (Email: tahmasebi@pgu.ac.ir). Department of Statistics, Persian Gulf University, Bushehr, Iran.
}

ISSN 2310-5070 (online) ISSN 2311-004X (print)

Copyright (C) 2020 International Academic Press 
where $\tilde{\Lambda}(x)=-\log F(x)$. Analogous to (2), Misagh et al. [12] proposed weighted cumulative residual entropy (WCRE) as

$$
\mathcal{E}^{w}(X)=\int_{0}^{+\infty} x \bar{F}(x) \Lambda(x) d x .
$$

Similarly, Misagh et al. [12] proposed weighted cumulative entropy (WCE) as

$$
\mathcal{C E}^{w}(X)=\int_{0}^{+\infty} x F(x) \tilde{\Lambda}(x) d x .
$$

Now, suppose that $X$ and $Y$ are two non-negative random variables with reliability functions $\bar{F}(x), \bar{G}(x)$, respectively. If $\bar{F}(x)$ is the actual survival function corresponding to the observations and $\bar{G}(x)$ is the survival function assigned by the experimenter, then Kumar and Taneja [11] defined the cumulative residual inaccuracy (CRI) based on $\bar{F}(x)$ and $\bar{G}(x)$ as follows:

$$
I(\bar{F}, \bar{G})=-\int_{0}^{+\infty} \bar{F}(x) \log \bar{G}(x) d x .
$$

In analogy with (6), a measure of cumulative past inaccuracy (CPI) associated with $F$ and $G$ is given by

$$
\tilde{I}(F, G)=-\int_{0}^{+\infty} F(x) \log G(x) d x
$$

Order statistics play an important role in problems such as industrial stress testing, meteorological analysis, hydrology, economics and other related fields. Different order statistics can be used in different applications; for example, the maximum is of interest in the study of floods and other meteorological phenomena while the minimum is often used in reliability and survival analysis, etc. For more details about order statistics and their applications, one may refer to [1]. Let $X_{1}, X_{2}, \ldots, X_{n}$ be a random sample of size $n$ from an absolutely continuous cumulative distribution function $F(x)$. If $X_{(1)} \leq X_{(2)} \leq \ldots \leq X_{(n)}$ represent the order statistics of the sample $X_{1}, X_{2}, \ldots, X_{n}$. Then the empirical measure of $F(x)$ is defined as

$$
\hat{F}_{n}(x)=\left\{\begin{array}{cc}
0, & x<X_{(1)}, \\
\frac{k}{n}, & X_{(k)} \leq x \leq X_{(k+1)}, \quad k=1,2, \ldots, n-1 \\
1, & x>X_{(k+1)} .
\end{array}\right.
$$

Recently Thapliyal and Taneja [21] have introduced the measure of residual inaccuracy of order statistics and proved a characterization result for it. Tahmasebi and Daneshi [19] and Tahmasebi et al. [20] have obtained some results of inaccuracy measures in record values. Eskandarzadeh et al. [5] have discussed the cumulative measure of inaccuracy in k-lower record values and studied characterization results of dynamic cumulative inaccuracy. Daneshi et al. [4] have proposed a weighted cumulative past (residual) inaccuracy of record values and studied its characterization results. The paper is organized as follows: In Section 2, we consider a measure of weighted cumulative residual inaccuracy (WCRI) between $\bar{F}_{X_{(1: n)}}$ and $\bar{F}$ and study its properties. In Section 3, we also propose the weighted cumulative past inaccuracy (WPCI) between $F_{X_{(n: n)}}$ and $F$ and obtain an estimator of cumulative inaccuracy using empirical approach .

\section{WCRI For Minimum of Order Statistics}

In this section, we propose the WCRI between $\bar{F}_{X_{(1: n)}}$ and $\bar{F}$ as follows:

$$
I^{w}\left(\bar{F}_{X_{(1: n)}}, \bar{F}\right)=-\int_{0}^{+\infty} x \bar{F}_{X_{(1: n)}}(x) \Lambda(x) d x=\frac{1}{n} \mathcal{E}^{w}\left(X_{(1: n)}\right),
$$


where

$$
\mathcal{E}^{w}\left(X_{(1: n)}\right)=n \int_{0}^{+\infty} x[\bar{F}(x)]^{n} \Lambda(x) d x .
$$

Hereafter we present some properties of $I^{w}\left(\bar{F}_{X_{(1: n)}}, \bar{F}\right)$.

\section{Proposition 1}

Let $X$ be an absolutely continuous non-negative random variable with $I\left(\bar{F}_{X_{(1: n)}}, \bar{F}\right)<\infty$, for $n \geq 1$. Then, we have

$$
\begin{aligned}
I^{w}\left(\bar{F}_{X_{(1: n)}}, \bar{F}\right)= & \int_{0}^{+\infty} x[\bar{F}(x)]^{n}\left(\int_{0}^{x} \frac{f(z)}{\bar{F}(z)} d z\right) d x \\
& =\int_{0}^{+\infty} \int_{z}^{+\infty} \lambda(z) x[\bar{F}(x)]^{n} d x d z
\end{aligned}
$$

where $\lambda($.$) is the failure rate function.$

\section{Proposition 2}

Let $X$ be an absolutely continuous non-negative random variable with $I^{w}\left(\bar{F}_{X_{(1: n)}}, \bar{F}\right)<\infty$, for $n \geq 1$. Then, we have

$$
I^{w}\left(\bar{F}_{X_{(1: n)}}, \bar{F}\right)=E\left(M_{(1: n)}^{w}(Z)(\bar{F}(Z))^{n-1}\right)
$$

where

$$
M_{(1: n)}^{w}(z)=\frac{1}{(\bar{F}(z))^{n}} \int_{z}^{+\infty} x(\bar{F}(x))^{n} d x
$$

is the weighted mean residual lifetime (wmrl) of $X_{(1: n)}$.

Proof

From (9), we have

$$
\begin{aligned}
I^{w}\left(\bar{F}_{X_{(1: n)}}, \bar{F}\right) & =\int_{0}^{+\infty} \int_{z}^{+\infty} \lambda(z) x[\bar{F}(x)]^{n} d x d z \\
& =\int_{0}^{+\infty} \frac{f(z)}{\bar{F}(z)} d z\left[\int_{z}^{+\infty} x[\bar{F}(x)]^{n} d x\right] \\
& =\int_{0}^{+\infty} \frac{f(z)}{\bar{F}(z)}[\bar{F}(z)]^{n} M_{(1: n)}^{w}(z) d z=\int_{0}^{+\infty} f(z)[\bar{F}(z)]^{n-1} M_{(1: n)}^{w}(z) d z
\end{aligned}
$$

So, the proof is complete.

Proposition 3

Let $a, b>0$. For $n=1,2, \ldots$ it holds that

$$
I^{w}\left(\bar{F}_{a X_{(1: n)}+b}, \bar{F}_{a X+b}\right)=a I^{w}\left(\bar{F}_{X_{(1: n)}}, \bar{F}\right) .
$$

The next propositions give some lower and upper bounds for $I^{w}\left(\bar{F}_{X_{(1: n)}}, \bar{F}\right)$.

Proposition 4

For a non-negative random variable $X$ and $n \geq 1$, it holds that

$$
I^{w}\left(\bar{F}_{X_{(1: n)}}, \bar{F}\right) \geq M_{(1: n)}^{w}(t)|\log \bar{F}(t)|[\bar{F}(t)]^{n},
$$

where $M_{(1: n)}^{w}(t)$ is the wmrl of $X_{(1: n)}$. 
Proof

The proof follows from Baratpour [2].

\section{Proposition 5}

Let $X$ be an absolutely continuous non-negative random variable with $I^{w}\left(\bar{F}_{X_{(1: n)}}, \bar{F}\right)<\infty$, for $n \geq 1$. Then, we have

$$
I^{w}\left(\bar{F}_{X_{(1: n)}}, \bar{F}\right) \geq \int_{0}^{+\infty} x(\bar{F}(x))^{n} F(x) d x .
$$

Proof

Recalling that $-\log \bar{F}(x) \geq F(x)$, the proof then finally follows.

\section{Proposition 6}

Let $X$ be an absolutely continuous non-negative random variable with $I^{w}\left(\bar{F}_{X_{(1: n)}}, \bar{F}\right)<\infty$, for $n \geq 1$. Then, we have

$$
I^{w}\left(\bar{F}_{X_{(1: n)}}, \bar{F}\right) \leq \mathcal{E}^{w}(X)
$$

Proof

Since $\bar{F}(x) \geq[\bar{F}(x)]^{n}, x \geq 0$, when $n \geq 1$, the proof then finally follows.

\section{Proposition 7}

If $X$ is IFRA (DFRA), then

$$
I^{w}\left(\bar{F}_{X_{(1: n)}}, \bar{F}\right) \leq(\geq) \mathbb{E}\left(X^{2}(\bar{F}(X))^{n-1}\right)
$$

Proof

Since $X$ is IFRA (DFRA), $\frac{\Lambda(x)}{x}$ is increasing (decreasing) with respect to $x>0$, which implies that

$$
\bar{F}(x) \Lambda(x) \leq(\geq) x f(x), \quad x>0 .
$$

By multiplying $x[\bar{F}(x)]^{n-1} \geq 0$ in (16) and then integrating, the result follows.

\section{Proposition 8}

Let $X$ be an absolutely continuous non-negative random variable with $I^{w}\left(\bar{F}_{X_{(1: n)}}, \bar{F}\right)<\infty$, for $n \geq 1$. Then, we have

$$
I^{w}\left(\bar{F}_{X_{(1: n)}}, \bar{F}\right)=\mathbb{E}\left[h^{w}(X)\right],
$$

where

$$
h^{w}(x)=\int_{0}^{x} z[-\log \bar{F}(z)][\bar{F}(z)]^{n-1} d z, \quad x \geq 0 .
$$

Proof

From (8) and using Fubini's theorem, we obtain

$$
\begin{aligned}
I^{w}\left(\bar{F}_{X_{(1: n)}}, \bar{F}\right) & =\int_{0}^{\infty} z[-\log \bar{F}(z)][\bar{F}(z)]^{n-1} \bar{F}(z) d z \\
& =\int_{0}^{\infty}\left[\int_{z}^{\infty} f(x) d x\right] z[\bar{F}(z)]^{n-1}[-\log \bar{F}(z)] d z \\
& =\int_{0}^{\infty} f(x)\left[\int_{0}^{x} z[\bar{F}(z)]^{n-1}[-\log \bar{F}(z)] d z\right] d x=\mathbb{E}\left[h^{w}(X)\right] .
\end{aligned}
$$


Proposition 9

Let $X$ and $Y$ be two non-negative random variables with reliability functions $\bar{F}(x), \bar{G}(x)$, respectively. If $X \leq^{i c x} Y$ , then

$$
I^{w}\left(\bar{F}_{X_{(1: n)}}, \bar{F}\right) \leq I^{w}\left(\bar{G}_{Y_{(1: n)}}, \bar{G}\right) .
$$

Proof

Since $h^{w}($.$) is an increasing convex function for n \geq 1$, it follows by Shaked and Shanthikumar [17] that $X \leq^{i c x} Y$ implies $h^{w}(X) \leq^{i c x} h^{w}(Y)$. By recalling the definition of increasing convex order and Proposition 8 proof is complete.

Proposition 10

Let $X$ and $Y$ be two non-negative random variables with survival function $\bar{F}(x)$ and $\bar{G}(x)$, respectively. If $X \leq{ }^{h r} Y$, then for $n=1,2, \ldots$, it holds that

$$
\frac{I^{w}\left(\bar{F}_{X_{(1: n)}}, \bar{F}\right)}{E(X)} \leq \frac{I^{w}\left(\bar{G}_{Y_{(1: n)}}, \bar{G}\right)}{E(Y)} .
$$

Proof

By noting that the function $h^{w}(x)=\int_{0}^{x} z[\bar{F}(z)]^{n-1}[-\log \bar{F}(z)] d z$ is an increasing convex function, under the assumption $X \leq^{h r} Y$, it follows by Shaked and Shanthikumar [17],

$$
\frac{E\left[h^{w}(X)\right]}{E(X)} \leq \frac{E\left[h^{w}(Y)\right]}{E(Y)}
$$

Hence, the proof is completed by recalling (17).

\section{Proposition 11}

(i) Let $X$ be a continuous random variable with survival function $\bar{F}($.$) that takes values in [0, b]$, with finite $b$. Then,

$$
I^{w}\left(\bar{F}_{X_{(1: n)}}, \bar{F}\right) \leq b I\left(\bar{F}_{X_{(1: n)}}, \bar{F}\right) .
$$

(ii) Let $X$ be a non-negative continuous random variable with survival function $\bar{F}($.$) that takes values in [a, \infty)$, with finite $a>0$. Then,

$$
I^{w}\left(\bar{F}_{X_{(1: n)}}, \bar{F}\right) \geq a I\left(\bar{F}_{X_{(1: n)}}, \bar{F}\right) .
$$

Assume that $X_{\theta}^{*}$ denotes a non-negative absolutely continuous random variable with the survival function $\bar{H}_{\theta}(x)=[\bar{F}(x)]^{\theta}, x \geq 0$. This model is known as a proportional hazards rate model. We now obtain the weighted cumulative residual measure of inaccuracy between $\bar{H}_{X_{(1: n)}}$ and $\bar{H}$ as follows:

$$
\begin{aligned}
I^{w}\left(\bar{H}_{X_{(1: n)}}, \bar{H}\right) & =-\int_{0}^{+\infty} x \bar{H}_{X_{(1: n)}}(x) \log (\bar{H}(x)) d x \\
& =-\theta \int_{0}^{+\infty} x(\bar{F}(x))^{n \theta} \log \bar{F}(x) d x
\end{aligned}
$$

Proposition 12

If $\theta \geq(\leq) 1$, then for any $n \geq 1$, we have

$$
I^{w}\left(\bar{H}_{X_{(1: n)}}, \bar{H}\right) \leq(\geq) \theta I^{w}\left(\bar{F}_{X_{(1: n)}}, \bar{F}\right) \leq(\geq) \theta \mathcal{E}^{w}(X) .
$$

Proof

Suppose that $\theta \geq(\leq) 1$, then it is clear $[\bar{F}(x)]^{\theta} \leq(\geq) \bar{F}(x)$, and hence (18) yields

$$
I^{w}\left(\bar{H}_{X_{(1: n)}}, \bar{H}\right) \leq(\geq) \theta \mathcal{E}^{w}(X) .
$$


Theorem 13

$I^{w}\left(\bar{F}_{X_{(1: n)}}, \bar{F}\right)=0$, if and only if, $X$ is degenerate.

Proof

Suppose $X$ is degenerate at point $a$, obviously by definition of degenerate function and definition of $I^{w}\left(\bar{F}_{X_{(1: n)}}, \bar{F}\right)$, we have $I^{w}\left(\bar{F}_{X_{(1: n)}}, \bar{F}\right)=0$.

Now, suppose that $I^{w}\left(\bar{F}_{X_{(1: n)}}, \bar{F}\right)=0$, i.e.

$$
-\int_{0}^{\infty} x[\bar{F}(x)]^{n} \log \bar{F}(x) d x=0 .
$$

Then, by noting that integrand function of (19) is non-negative, we conclude that $-x[\bar{F}(x)]^{n} \log \bar{F}(x)=0$, for almost all $x \in \mathbb{R}^{+}$. Thus, $\bar{F}(x)=0$ or 1 , for almost all $x \in \mathbb{R}^{+}$.

Recently, Cali et al. [3] introduced the generalized CPI of order $m$ defined as

$$
I_{m}(F, G)=\frac{1}{m !} \int_{0}^{+\infty} F(x)[-\log G(x)]^{m} d x .
$$

In analogy with the measure defined in (20), we now introduce the weighted generalized CRI (WGCRI) of order $m$ defined as

$$
I_{m}^{w}(\bar{F}, \bar{G})=\frac{1}{m !} \int_{0}^{+\infty} x \bar{F}(x)[-\log \bar{G}(x)]^{m} d x .
$$

Remark 1

Let $X$ be a non-negative absolutely continuous random variable with cdf $F$. Then, the WGCRI of order $m$ between $\bar{F}_{X_{(1: n)}}$ and $F$ is

$$
\begin{aligned}
I_{m}^{w}\left(\bar{F}_{X_{(1: n)}}, \bar{F}\right) & =\frac{1}{m !} \int_{0}^{\infty} x[\bar{F}(x)]^{n}[-\log \bar{F}(x)]^{m} d x \\
& =\frac{1}{n^{m}} \mathcal{E}_{m}^{w}\left(X_{(1: n)}\right),
\end{aligned}
$$

where

$$
\mathcal{E}_{m}^{w}(X)=\int_{0}^{\infty} x \frac{[-\log \bar{F}(x)]^{m}}{m !} \bar{F}(x) d x,
$$

is a weighted generalized cumulative residual entropy (WGCRE) which introduced by Kayal [9].

\section{Remark 2}

In analogy with (8), a measure of WCRI associated with $\bar{F}$ and $\bar{F}_{X_{(1: n)}}$ is given by

$$
I^{w}\left(\bar{F}, \bar{F}_{X_{(1: n)}}\right)=-\int_{0}^{+\infty} x \bar{F}(x) \log \left(\bar{F}_{X_{(1: n)}}(x)\right) d x=n \mathcal{E}^{w}(X) .
$$

In the remainder of this section, we study dynamic version of $I^{w}\left(\bar{F}_{X_{(1: n)}}, \bar{F}\right)$. Let $X$ be the lifetime of a system under condition that the system has survived up to age $t$. Analogously, we can also consider the dynamic version of $I^{w}\left(\bar{F}_{X_{(1: n)}}, \bar{F}\right)$ as

$$
\begin{aligned}
I^{w}\left(\bar{F}_{X_{(1: n)}}, \bar{F} ; t\right) & =-\int_{t}^{+\infty} x \frac{\bar{F}_{X_{(1: n)}}(x)}{\bar{F}_{X_{(1: n)}}(t)} \log \left(\frac{\bar{F}(x)}{\bar{F}(t)}\right) d x \\
& =\log \bar{F}(t) M_{(1: n)}^{w}(t)-\int_{t}^{+\infty} x \frac{\bar{F}_{X_{(1: n)}}(x)}{\bar{F}_{X_{(1: n)}}(t)} \log (\bar{F}(x)) d x
\end{aligned}
$$




$$
=\log \bar{F}(t) M_{(1: n)}^{w}(t)-\frac{1}{(\bar{F}(t))^{n}} \int_{t}^{+\infty} x(\bar{F}(x))^{n} \log \bar{F}(x) d x .
$$

Note that $\lim _{t \rightarrow 0} I^{w}\left(\bar{F}_{X_{(1: n)}}, \bar{F} ; t\right)=I^{w}\left(\bar{F}_{X_{(1: n)}}, \bar{F}\right)$. Since $\log \bar{F}(t) \leq 0$ for $t \geq 0$, we have

$$
\begin{aligned}
I^{w}\left(\bar{F}_{X_{(1: n)}}, \bar{F} ; t\right) & \leq-\frac{1}{(\bar{F}(t))^{n}} \int_{t}^{+\infty} x(\bar{F}(x))^{n} \log \bar{F}(x) d x \\
& \leq-\frac{1}{(\bar{F}(t))^{n}} \int_{0}^{+\infty} x(\bar{F}(x))^{n} \log \bar{F}(x) d x=\frac{I^{w}\left(\bar{F}_{X_{(1: n)}}, \bar{F}\right)}{(\bar{F}(t))^{n}} .
\end{aligned}
$$

Theorem 14

Let $X$ be a non-negative continuous random variable with distribution function $F($.$) . Let the weighted dynamic$ cumulative inaccuracy of the 1 th order statistics denoted by $I^{w}\left(\bar{F}_{X_{(1: n)}}, \bar{F} ; t\right)<\infty, t \geq 0$. Then $I^{w}\left(\bar{F}_{X_{(1: n)}}, \bar{F} ; t\right)$ characterizes the distribution function.

Proof

From (24) we have

$$
I^{w}\left(\bar{F}_{X_{(1: n)}}, \bar{F} ; t\right)=\log \bar{F}(t) M_{(1: n)}^{w}(t)-\frac{1}{(\bar{F}(t))^{n}} \int_{t}^{+\infty} x(\bar{F}(x))^{n} \log \bar{F}(x) d x .
$$

Differentiating both side of (25) with respect to $t$ we obtain:

$$
\begin{aligned}
\frac{\partial}{\partial t}\left[I^{w}\left(\bar{F}_{X_{(1: n)}}, \bar{F} ; t\right)\right] & =-\lambda_{F}(t) M_{(1: n)}^{w}(t)+n \lambda_{F}(t) I^{w}\left(\bar{F}_{X_{(1: n)}}, \bar{F} ; t\right) \\
& =\lambda_{F}(t)\left[n I^{w}\left(\bar{F}_{X_{(1: n)}}, \bar{F} ; t\right)-M_{(1: n)}^{w}(t)\right] .
\end{aligned}
$$

Taking derivative with respect to $t$ again we get

$$
\dot{\lambda}_{F}(t)=\frac{\left(\lambda_{F}(t)\right)^{2}\left(n \lambda_{F}(t) M_{(1: n)}^{w}(t)+n \frac{\partial}{\partial t} I^{w}\left(\bar{F}_{X_{(1: n)}}, \bar{F} ; t\right)-t\right)}{\frac{\partial}{\partial t} I^{w}\left(\bar{F}_{X_{(1: n)}}, \bar{F} ; t\right)} .
$$

Suppose that there are two functions $F$ and $F^{*}$ such that

$$
I^{w}\left(\bar{F}_{X_{(1: n)}}, \bar{F} ; t\right)=I^{w}\left(\bar{F}_{X_{(1: n)}}^{*}, \bar{F}^{*} ; t\right)=z(t) .
$$

Then for all $t$, from (26) we get

$$
\hat{\lambda}_{F}(t)=\varphi\left(t, \lambda_{F}(t)\right), \quad \hat{\lambda}_{F^{*}}(t)=\varphi\left(t, \lambda_{F^{*}}(t)\right),
$$

where

$$
\varphi(t, y)=\frac{y^{2}[n y s(t)+n \dot{z}(t)-t]}{\dot{z}(t)}
$$

and $s(t)=M_{(1: n)}^{w}(t)$. By using Theorem 3.2 and Lemma 3.3 of Gupta and Kirmani [8], we have, $\lambda_{F}(t)=\lambda_{F^{*}}(t)$, for all $t$. Since the hazard rate function characterizes the distribution function uniquely, we complete the proof.

\section{Proposition 15}

If $X_{(1)} \leq X_{(2)} \leq \ldots \leq X_{(n)}$ represent the order statistics of the sample $X_{1}, X_{2}, \ldots, X_{n}$. Then, the empirical measure of $I^{w}\left(\bar{F}_{X_{(1: n)}}, \bar{F}\right)$ is obtained as

$$
\hat{I}^{w}\left(\bar{F}_{X_{(1: n)}}, \bar{F}\right)=-\int_{0}^{+\infty} x\left[\hat{\bar{F}}_{n}(x)\right]^{n} \log \hat{\bar{F}}_{n}(x) d x
$$




$$
\begin{aligned}
& =-\sum_{k=1}^{n-1} \int_{X_{(k)}}^{X_{(k+1)}} x\left(1-\frac{k}{n}\right)^{n} \log \left(1-\frac{k}{n}\right) d x \\
& =-\sum_{k=1}^{n-1} U_{k}\left(1-\frac{k}{n}\right)^{n} \log \left(1-\frac{k}{n}\right)
\end{aligned}
$$

where $U_{k}=\frac{X_{(k+1)}^{2}-X_{(k)}^{2}}{2}, k=1,2, \ldots, n-1$.

Theorem 16

Let $\mathrm{X}$ be a absolutely continue non-negative random variable whit $I^{w}\left(\bar{F}_{X_{(1: n)}}, \bar{F}\right)<\infty$, for all $n \geq 1$. Then we have

$$
\hat{I}^{w}\left(\bar{F}_{X_{(1: n)}}, \bar{F}\right) \longrightarrow I^{w}\left(\bar{F}_{X_{(1: n)}}, \bar{F}\right) \text { a.s. }
$$

Proof

From (27) we have

$$
\begin{aligned}
\hat{I}^{w}\left(\bar{F}_{X_{(1: n)}}, \bar{F}\right) & =\int_{0}^{\infty} x\left(-\log \hat{\bar{F}}_{n}(x)\right)\left(\hat{\bar{F}}_{n}(x)\right)^{n} d x \\
& =\int_{0}^{1} x\left(-\log \hat{\bar{F}}_{n}(x)\right)\left(\hat{\bar{F}}_{n}(x)\right)^{n} d x+\int_{1}^{\infty} x\left(-\log \hat{\bar{F}}_{n}(x)\right)\left(\hat{\bar{F}}_{n}(x)\right)^{n} d x \\
& =: W_{1}+W_{2},
\end{aligned}
$$

where

$$
\begin{gathered}
W_{1}=\int_{0}^{1} x\left(-\log \hat{\bar{F}}_{n}(x)\right)\left(\hat{\bar{F}}_{n}(x)\right)^{n} d x, \\
W_{2}=\int_{1}^{\infty} x\left(-\log \hat{\bar{F}}_{n}(x)\right)\left(\hat{\bar{F}}_{n}(x)\right)^{n} d x .
\end{gathered}
$$

Using dominated convergence theorem (DCT) and Glivenko-Cantelli, we have

$$
\int_{0}^{1} x\left(-\log \hat{\bar{F}}_{n}(x)\right)\left(\hat{\bar{F}}_{n}(x)\right)^{n} d x \longrightarrow \int_{0}^{1} x(-\log \bar{F}(x))(\bar{F}(x))^{n} d x \quad \text { as } m \rightarrow \infty .
$$

It follows that

$$
x^{p} \hat{\bar{F}}_{n}(x) \leq \frac{1}{n} \sum_{i=1}^{n} X_{i}^{p} .
$$

Morever, by using $\operatorname{SLLN}, \quad \frac{1}{n} \sum_{i=1}^{n} X_{i}^{p} \longrightarrow \mathbb{E}\left(X^{p}\right) \quad$ and $\quad \sup _{n}\left(\frac{1}{n} \sum_{i=1}^{n} X_{i}^{p}\right)<\infty, \quad$ then $\quad \hat{\bar{F}}_{n}(x) \leq$ $x^{-p}\left(\sup _{n}\left(\frac{1}{n} \sum_{i=1}^{n} X_{i}^{p}\right)\right)=C x^{-p}$. Now applying the DCT we have

$$
\lim _{n \rightarrow \infty} W_{2}=\int_{1}^{\infty} x(-\log \bar{F}(x))(\bar{F}(x))^{n} d x .
$$

Finally by using (28) the result follows.

\section{WCPI For Maximum of Order Statistics}

We consider the WCPI between $F_{X_{(n: n)}}$ and $F$ as follows:

$$
\tilde{I}^{w}\left(F_{X_{(n: n)}}, F\right)=-\int_{0}^{+\infty} x F_{X_{(n: n)}}(x) \log (F(x)) d x=\frac{1}{n} \mathcal{C} \mathcal{E}^{w}\left(X_{(n: n)}\right),
$$


where

$$
\mathcal{C E}^{w}\left(X_{(n: n)}\right)=n \int_{0}^{+\infty} x[F(x)]^{n} \tilde{\Lambda}(x) d x .
$$

Hereafter we consider some properties of $\tilde{I}^{w}\left(F_{X_{(n: n)}}, F\right)$.

\section{Proposition 17}

Let $X$ be an absolutely continuous non-negative random variable with $\tilde{I}^{w}\left(F_{X_{(n: n)}}, F\right)<\infty$, for $n \geq 1$. Then, we have

$$
\begin{array}{r}
\tilde{I}^{w}\left(F_{X_{(n: n)}}, F\right)=\int_{0}^{+\infty} x[F(x)]^{n}\left(\int_{x}^{\infty} \frac{f(z)}{F(z)} d z\right) x d x \\
=\int_{0}^{+\infty} \int_{0}^{z} \tilde{\lambda}(z) x[F(x)]^{n} d x d z
\end{array}
$$

where $\tilde{\lambda}($.$) is the reversed failure rate function.$

\section{Proposition 18}

Let $X$ be an absolutely continuous non-negative random variable with $\tilde{I}^{w}\left(F_{X_{(n: n)}}, F\right)<\infty$, for $n \geq 1$. Then, we have

$$
\tilde{I}^{w}\left(F_{X_{(n: n)}}, F\right)=E\left(\tilde{M}_{(n: n)}^{w}(Z)(F(Z))^{n-1}\right),
$$

where

$$
\tilde{M}_{(n: n)}^{w}(z)=\frac{1}{(F(z))^{n}} \int_{0}^{z} x(F(x))^{n} d x
$$

is the weighted mean inactivity time (WMIT) of $X_{(n: n)}$.

Proof

By (32), we obtain

$$
\begin{aligned}
\tilde{I}^{w}\left(F_{X_{(n: n)}}, F\right) & =\int_{0}^{+\infty} \int_{0}^{z} \tilde{\lambda}(z) x[F(x)]^{n} d x d z \\
& =\int_{0}^{+\infty} \frac{f(z)}{F(z)} d z\left[\int_{0}^{z} x[F(x)]^{n} d x\right] \\
& =\int_{0}^{+\infty} \frac{f(z)}{F(z)}[F(z)]^{n} \tilde{M}_{(n: n)}^{w}(z) d z=\int_{0}^{+\infty} f(z)[F(z)]^{n-1} \tilde{M}_{(n: n)}^{w}(z) d z .
\end{aligned}
$$

Thus, the proof is complete.

\section{Proposition 19}

Let $a, b>0$. For $n=1,2, \ldots$ it holds that

$$
\tilde{I}^{w}\left(F_{a X_{(n: n)}+b}, F_{a X+b}\right)=a \tilde{I}^{w}\left(F_{X_{(n: n)}}, F\right) .
$$

The next propositions give some lower and upper bounds for $\tilde{I}^{w}\left(F_{X_{(n: n)}}, F\right)$.

\section{Proposition 20}

For a non-negative random variable $X$ and $n \geq 1$, it holds that

$$
\tilde{I}^{w}\left(F_{X_{(n: n)}}, F\right) \geq \tilde{M}_{(n: n)}^{w}(t)|\log F(t)|[F(t)]^{n},
$$

where $\tilde{M}_{(n: n)}(t)$ is the WMIT of $X_{(n: n)}$. 
Proof

The proof follows from Baratpour [2].

\section{Proposition 21}

Let $X$ be an absolutely continuous non-negative random variable with $\tilde{I}^{w}\left(F_{X_{(n: n)}}, F\right)<\infty$, for $n \geq 1$. Then, we have

$$
\tilde{I}^{w}\left(F_{X_{(n: n)}}, F\right) \geq \int_{0}^{+\infty} x(F(x))^{n} \bar{F}(x) d x
$$

Proof

Recalling that $-\log F(x) \geq \bar{F}(x)$, the proof then finally follows.

\section{Proposition 22}

Let $X$ be an absolutely continuous non-negative random variable with $\tilde{I}^{w}\left(F_{X_{(n: n)}}, F\right)<\infty$, for $n \geq 1$. Then, we have

$$
\tilde{I}^{w}\left(F_{X_{(n: n)}}, F\right) \leq \mathcal{C E}^{w}(X)
$$

Proof

Since $F(x) \geq[F(x)]^{n}, x \geq 0$, when $n \geq 1$, the proof then finally follows.

\section{Proposition 23}

If $X$ is DRFRA, then

$$
\tilde{I}^{w}\left(F_{X_{(n: n)}}, F\right) \leq \mathbb{E}\left(X^{2}(F(X))^{n-1}\right) .
$$

Proof

Since $X$ is DRFRA, $\frac{\tilde{\Lambda}(x)}{x}$ is decreasing with respect to $x>0$, which implies that

$$
F(x) \tilde{\Lambda}(x) \leq x f(x), \quad x>0 .
$$

By multiplying $x[F(x)]^{n-1} \geq 0$ in (39) and then integrating, the result follows.

Proposition 24

Let $X$ be an absolutely continuous non-negative random variable with $\tilde{I}^{w}\left(F_{X_{(n: n)}}, F\right)<\infty$, for $n \geq 1$. Then, we have

$$
\tilde{I}^{w}\left(F_{X_{(n: n)}}, F\right)=\mathbb{E}\left[\tilde{h}^{w}(X)\right]
$$

where

$$
\tilde{h}^{w}(x)=\int_{x}^{\infty} z[-\log F(z)][F(z)]^{n-1} d z, \quad x \geq 0 .
$$

Proof

From (31) and using Fubini's theorem, we obtain

$$
\begin{aligned}
\tilde{I}^{w}\left(F_{X_{(n: n)}}, F\right) & =\int_{0}^{\infty} z[-\log F(z)][F(z)]^{n-1} F(z) d z \\
& =\int_{0}^{\infty}\left[\int_{0}^{z} f(x) d x\right] z[F(z)]^{n-1}[-\log F(z)] d z \\
& =\int_{0}^{\infty} f(x)\left[\int_{x}^{\infty} z[F(z)]^{n-1}[-\log F(z)] d z\right] d x=\mathbb{E}\left[\tilde{h}^{w}(X)\right] .
\end{aligned}
$$


Proposition 25

Let $X$ and $Y$ be two non-negative random variables with reliability functions $\bar{F}(x), \bar{G}(x)$, respectively. If $X \leq{ }^{i c x} Y$ , then

$$
\tilde{I}^{w}\left(F_{X_{(n: n)}}, F\right) \leq \tilde{I}^{w}\left(G_{Y_{(n: n)}}, G\right) .
$$

Proof

Since $\tilde{h}^{w}($.$) is an increasing convex function for n \geq 1$, it follows by Shaked and Shanthikumar [17] that $X \leq^{i c x} Y$ implies $\tilde{h}^{w}(X) \leq^{i c x} \tilde{h}^{w}(Y)$. By recalling the definition of increasing convex order and Proposition 24 proof is complete.

Proposition 26

Let $X$ and $Y$ be two non-negative random variables with survival function $\bar{F}(x)$ and $\bar{G}(x)$, respectively. If $X \leq^{h r} Y$, then for $n=1,2, \ldots$, it holds that

$$
\frac{\tilde{I}^{w}\left(F_{X_{(n: n)}}, F\right)}{E(X)} \leq \frac{\tilde{I}^{w}\left(G_{Y_{(n: n)}}, G\right)}{E(Y)} .
$$

Proof

By noting that the function $\tilde{h}^{w}(x)=\int_{x}^{\infty} z[F(z)]^{n-1}[-\log F(z)] d z$ is an increasing convex function, under the assumption $X \leq{ }^{h r} Y$, it follows by Shaked and Shanthikumar [17],

$$
\frac{E\left[\tilde{h}^{w}(X)\right]}{E(X)} \leq \frac{E\left[\tilde{h}^{w}(Y)\right]}{E(Y)}
$$

Hence, the proof is completed by recalling (40).

Proposition 27

(i) Let $X$ be a continuous random variable with survival function $\bar{F}($.$) that takes values in [0, b]$, with finite $b$. Then,

$$
\tilde{I}^{w}\left(F_{X_{(n: n)}}, F\right) \leq b \tilde{I}\left(F_{X_{(n: n)}}, F\right) .
$$

(ii) Let $X$ be a non-negative continuous random variable with survival function $\bar{F}($.$) that takes values in [a, \infty)$, with finite $a>0$. Then,

$$
\tilde{I}^{w}\left(F_{X_{(n: n)}}, F\right) \geq a \tilde{I}\left(F_{X_{(n: n)}}, F\right) .
$$

Assume that $X_{\theta}^{*}$ denotes a non-negative absolutely continuous random variable with the distribution function $H_{\theta}(x)=[F(x)]^{\theta}, x \geq 0$. This model is known as a proportional hazards rate model. We now obtain the weighted cumulative past measure of inaccuracy between $H_{X_{(n: n)}}$ and $H$ as follows:

$$
\begin{aligned}
\tilde{I}^{w}\left(H_{X_{(n: n)}}, H\right) & =-\int_{0}^{+\infty} x H_{X_{(n: n)}}(x) \log (H(x)) d x \\
& =-\theta \int_{0}^{+\infty} x(F(x))^{n \theta} \log F(x) d x .
\end{aligned}
$$

Proposition 28

If $\theta \geq(\leq) 1$, then for any $n \geq 1$, we have

$$
\tilde{I}^{w}\left(H_{X_{(n: n)}}, H\right) \leq(\geq) \theta \tilde{I}^{w}\left(F_{X_{(n: n)}}, F\right) \leq(\geq) \theta \mathcal{C} \mathcal{E}^{w}(X) .
$$

Proof

Suppose that $\theta \geq(\leq) 1$, then it is clear $[F(x)]^{\theta} \leq(\geq) F(x)$, and hence (41) yields

$$
\tilde{I}^{w}\left(H_{X_{(n: n)}}, H\right) \leq(\geq) \theta \mathcal{C} \mathcal{E}^{w}(X) .
$$


Theorem 29

$\tilde{I}^{w}\left(F_{X_{(n: n)}}, F\right)=0$, if and only if, $\mathrm{X}$ is degenerate.

Proof

Suppose $X$ is degenerate at point $a$, obviously by definition of degenerate function and definition of $\tilde{I}^{w}\left(F_{X_{(n: n)}}, F\right)$, we have $\tilde{I}^{w}\left(F_{X_{(n: n)}}, F\right)=0$.

Now, suppose that $\tilde{I}^{w}\left(F_{X_{(n: n)}}, F\right)=0$, i.e.

$$
-\int_{0}^{\infty} x[F(x)]^{n} \log F(x) d x=0 .
$$

Then, by noting that integrand function of (42) is non-negative, we conclude that $-x[F(x)]^{n} \log F(x)=0$, for almost all $x \in \mathbb{R}^{+}$. Thus, $F(x)=0$ or 1 , for almost all $x \in \mathbb{R}^{+}$.

Proposition 30

Let $\mathrm{X}$ be an absolutely continuous random variable with $\tilde{I}^{w}\left(F_{X_{(n: n)}}, F\right)<\infty$, for $n \geq 1$. Then, we have

$$
\tilde{I}^{w}\left(F_{X_{(n: n)}}, F\right)=\int_{0}^{1} F_{X}^{-1}(u) \frac{\psi_{n}(u)}{f_{X}\left(F_{X}^{-1}(u)\right)} d u,
$$

where $\psi_{n}(u)=-u^{n} \log u$, for $0<u<1$. Note that $\psi_{n}(0)=\psi_{n}(1)=0$.

Recently, Cali et al. [3] introduced the generalized CPI of order $m$ defined as

$$
I_{m}(F, G)=\frac{1}{m !} \int_{0}^{+\infty} F(x)[-\log G(x)]^{m} d x .
$$

In analogy with the measure defined in (43), we now introduce the weighted generalized CPI (WGCPI) of order $m$ defined as

$$
I_{m}^{w}(F, G)=\frac{1}{m !} \int_{0}^{+\infty} x F(x)[-\log G(x)]^{m} d x .
$$

Remark 3

Let $X$ be a non-negative absolutely continuous random variable with cdf $F$. Then, the WGCPI of order $m$ between $F_{X_{(n: n)}}$ and $F$ is

$$
\begin{aligned}
\tilde{I}_{m}^{w}\left(F_{X_{(n: n)}}, F\right) & =\frac{1}{m !} \int_{0}^{\infty} x[F(x)]^{n}[-\log F(x)]^{m} d x \\
& =\frac{1}{n^{m}} \mathcal{C} \mathcal{E}_{m}^{w}\left(X_{(n: n)}\right),
\end{aligned}
$$

where

$$
\mathcal{C E}_{m}^{w}(X)=\int_{0}^{\infty} x \frac{[-\log F(x)]^{m}}{m !} F(x) d x,
$$

is a weighted generalized cumulative entropy (WGCE) which introduced by Kayal and Moharana [10].

\section{Remark 4}

In analogy with (31), a measure of WCPI associated with $F$ and $F_{X_{(n: n)}}$ is given by

$$
\tilde{I}^{w}\left(F, F_{X_{(n: n)}}\right)=-\int_{0}^{+\infty} x F(x) \log \left(F_{X_{(n: n)}}(x)\right) d x=n \mathcal{C E}^{w}(X) .
$$


In the remainder of this section, we study dynamic version of $\tilde{I}^{w}\left(F_{X_{(n: n)}}, F\right)$. If a system that begins to work at time 0 is observed only at deterministic inspection times, and is found to be down at time t, then we consider a dynamic version of $\tilde{I}^{w}\left(F_{X_{(n: n)}}, F\right)$ as

$$
\begin{aligned}
\tilde{I}^{w}\left(F_{X_{(n: n)}}, F ; t\right) & =-\int_{0}^{t} x \frac{F_{X_{(n: n)}}(x)}{F_{X_{(n: n)}}(t)} \log \left(\frac{F(x)}{F(t)}\right) d x \\
& =\log F(t) \tilde{M}_{(n: n)}^{w}(t)-\int_{0}^{t} x \frac{F_{X_{(n: n)}}(x)}{F_{X_{(n: n)}}(t)} \log (F(x)) d x \\
& =\log F(t) \tilde{M}_{(n: n)}^{w}(t)-\frac{1}{(F(t))^{n}} \int_{0}^{t} x(F(x))^{n} \log F(x) d x .
\end{aligned}
$$

Note that $\lim _{t \rightarrow 0} \tilde{I}^{w}\left(F_{X_{(n: n)}}, F ; t\right)=\tilde{I}^{w}\left(F_{X_{(n: n)}}, F\right)$. Since $\log F(t) \leq 0$ for $t \geq 0$, we have

$$
\begin{aligned}
\tilde{I}^{w}\left(F_{X_{(n: n)}}, F ; t\right) & \leq-\frac{1}{(F(t))^{n}} \int_{0}^{t} x(F(x))^{n} \log F(x) d x \\
& \leq-\frac{1}{(F(t))^{n}} \int_{0}^{+\infty} x(F(x))^{n} \log F(x) d x=\frac{\tilde{I}^{w}\left(F_{X_{(n: n)}}, F\right)}{(F(t))^{n}}
\end{aligned}
$$

\section{Theorem 31}

Let $X$ be a non-negative continuous random variable with distribution function $F($.$) . Let the weighted dynamic$ cumulative inaccuracy of the nth order statistics denoted by $\tilde{I}^{w}\left(F_{X_{(n: n)}}, F ; t\right)<\infty, t \geq 0$. Then $\tilde{I}^{w}\left(F_{X_{(n: n)}}, F ; t\right)$ characterizes the distribution function.

Proof

From (47) we have

$$
\tilde{I}^{w}\left(F_{X_{(n: n)}}, F ; t\right)=\log F(t) \tilde{M}_{(n: n)}^{w}(t)-\frac{1}{(F(t))^{n}} \int_{0}^{t} x(F(x))^{n} \log F(x) .
$$

Differentiating both side of (48) with respect to $t$ we obtain:

$$
\begin{aligned}
\frac{\partial}{\partial t}\left[\tilde{I}^{w}\left(F_{X_{(n: n)}}, F ; t\right)\right] & =-\tilde{\lambda}_{F}(t) \tilde{M}_{(n: n)}^{w}(t)-n \tilde{\lambda}_{F}(t) \tilde{I}^{w}\left(F_{X_{(n: n)}}, F ; t\right) \\
& =-\tilde{\lambda}_{F}(t)\left[\tilde{M}_{(n: n)}^{w}(t)-n \tilde{I}^{w}\left(F_{X_{(n: n)}}, F ; t\right)\right]
\end{aligned}
$$

Taking derivative with respect to $t$ again we get

$$
\tilde{\tilde{\lambda}}_{F}(t)=\frac{\left(\tilde{\lambda}_{F}(t)\right)^{2}\left(n \tilde{\lambda}_{F}(t) \tilde{M}_{(n: n)}^{w}(t)+n \frac{\partial}{\partial t} \tilde{I}^{w}\left(F_{X_{(n: n)}}, F ; t\right)-t\right)}{\frac{\partial}{\partial t} \tilde{I}^{w}\left(F_{X_{(n: n)}}, F ; t\right)} .
$$

Suppose that there are two functions $F$ and $F^{*}$ such that

$$
\tilde{I}^{w}\left(F_{X_{(n: n)}}, F ; t\right)=\tilde{I}^{w}\left(F_{X_{(n: n)}}^{*}, F^{*} ; t\right)=z(t) .
$$

Then for all $t$, from (49) we get

$$
\dot{\tilde{\lambda}}_{F}(t)=\varphi\left(t, \lambda_{F}(t)\right), \quad \dot{\tilde{\lambda}}_{F^{*}}(t)=\varphi\left(t, \lambda_{F^{*}}(t)\right),
$$


where

$$
\varphi(t, y)=\frac{y^{2}[n y s(t)+n \dot{z}(t)-t]}{z(t)}
$$

and $\tilde{s}(t)=\tilde{M}_{(n: n)}^{w}(t)$. By using Theorem 3.2 and Lemma 3.3 of Gupta and Kirmani [8], we have, $\lambda_{F}(t)=\lambda_{F^{*}}(t)$, for all $t$. Since the hazard rate function characterizes the distribution function uniquely, we complete the proof.

\section{Proposition 32}

If $X_{(1)} \leq X_{(2)} \leq \ldots \leq X_{(n)}$ denote the order statistics of the sample $X_{1}, X_{2}, \ldots, X_{n}$. Then, the empirical measure of $\tilde{I}^{w}\left(F_{X_{(n: n)}}, F\right)$ is given by

$$
\begin{aligned}
\hat{\tilde{I}}^{w}\left(F_{X_{(n: n)}}, F\right) & =-\int_{0}^{+\infty} x\left[\hat{F}_{n}(x)\right]^{n} \log \hat{F}_{n}(x) d x \\
& =-\sum_{k=1}^{n-1} \int_{X_{(k)}}^{X_{(k+1)}} x\left(\frac{k}{n}\right)^{n} \log \left(\frac{k}{n}\right) d x \\
& =\frac{-1}{n^{n}} \sum_{k=1}^{n-1} k^{n} U_{k} \log \left(\frac{k}{n}\right)
\end{aligned}
$$

where $U_{k}=\frac{X_{(k+1)}^{2}-X_{(k)}^{2}}{2}, k=1,2, \ldots, n-1$.

Example 1

Consider the random sample $X_{1}, X_{2}, \ldots, X_{n}$ from a Weibull distribution with density function

$$
f(x)=2 \lambda \exp \left(-\lambda x^{2}\right) .
$$

Then $Y_{k}=X_{k}^{2}$ has an exponential distribution with mean $\frac{1}{\lambda}$. In this case, the sample spacings $2 U_{k}=X_{(k+1)}^{2}-X_{(k)}^{2}$ are independent and exponentially distributed with mean $\frac{1}{\lambda(n-k)}$ (for more details see Pyke [14]). Now from (50) we obtain

$$
\mathbb{E}\left[\hat{\tilde{I}}^{w}\left(F_{X_{(n: n)}}, F\right)\right]=\frac{-1}{n^{n}} \sum_{k=1}^{n-1} \frac{k^{n}}{2 \lambda(n-k)} \log \frac{k}{n},
$$

and

$$
\operatorname{Var}\left[\hat{\tilde{I}}^{w}\left(F_{X_{(n: n)}}, F\right)\right]=\frac{1}{n^{2 n}} \sum_{k=1}^{n-1} \frac{k^{2 n}}{4 \lambda^{2}(n-k)^{2}}\left(\log \frac{k}{n}\right)^{2} .
$$

We have computed the values of $\mathbb{E}\left[\hat{\tilde{I}}^{w}\left(F_{X_{(n: n)}}, F\right)\right]$ and $\operatorname{Var}\left[\hat{\tilde{I}}^{w}\left(F_{X_{(n: n)}}, F\right)\right]$ for sample sizes $n=10,15,20$ and $\lambda=0.5,1,2$ in Table 1 . We can easily see that $\mathbb{E}\left[\hat{\tilde{I}}^{w}\left(F_{X_{(n: n)}}, F\right)\right]$ is decreasing in $n$. Also, we consider that $\lim _{n \rightarrow \infty} \operatorname{Var}\left[\hat{\tilde{I}}^{w}\left(F_{X_{(n: n)}}, F\right)\right]=0$.

\section{Example 2}

Let $X_{1}, X_{2}, \ldots, X_{n}$ be a random sample from a population with pdf $f(x)=2 x, 0<x<1$. Then the sample spacings $2 U_{k}$ are independent of beta distribution with parameters 1 and $n$ (for more details see Pyke [14]). Now from (50) we obtain

$$
\mathbb{E}\left[\hat{\tilde{I}}^{w}\left(F_{X_{(n: n)}}, F\right)\right]=\frac{-1}{n^{n}} \sum_{k=1}^{n-1} \frac{k^{n}}{2(n+1)} \log \frac{k}{n},
$$

and

$$
\operatorname{Var}\left[\hat{\tilde{I}}^{w}\left(F_{X_{(n: n)}}, F\right)\right]=\frac{1}{n^{2 n-1}} \sum_{k=1}^{n-1} \frac{k^{n}}{4(n+1)^{2}(n+2)}\left(\log \frac{k}{n}\right)^{2} .
$$


We have computed the values of $\mathbb{E}\left[\hat{\tilde{I}}^{w}\left(F_{X_{(n: n)}}, F\right)\right]$ and $\operatorname{Var}\left[\hat{\tilde{I}}^{w}\left(F_{X_{(n: n)}}, F\right)\right]$ for sample sizes $n=10,15,20$ in Table 2. We can easily see that $\mathbb{E}\left[\hat{\tilde{I}}^{w}\left(F_{X_{(n: n)}}, F\right)\right]$ is decreasing in $n$. Also, we consider that $\lim _{n \rightarrow \infty} \operatorname{Var}\left[\hat{\tilde{I}}^{w}\left(F_{X_{(n: n)}}, F\right)\right]=0$.

Table 1. Numerical values of $\mathbb{E}\left[\hat{\tilde{I}}^{w}\left(F_{X_{(n: n)}}, F\right)\right]$ and $\operatorname{Var}\left[\hat{\tilde{I}}^{w}\left(F_{X_{(n: n)}}, F\right)\right]$ for Weibull distribution.

\begin{tabular}{|c|ccc|ccc|}
\hline & \multicolumn{3}{|c|}{$\mathbb{E}\left[\hat{\tilde{I}}^{w}\left(F_{X_{(n: n)}}, F\right)\right]$} & \multicolumn{3}{c|}{$\operatorname{Var}\left[\hat{\tilde{I}}^{w}\left(F_{X_{(n: n)}}, F\right)\right]$} \\
\hline$n$ & $\lambda=0.5$ & $\lambda=1$ & $\lambda=2$ & $\lambda=0.5$ & $\lambda=1$ & $\lambda=2$ \\
\hline 10 & 0.0530 & 0.0265 & 0.0132 & 0.00150 & 0.00037 & 0.00009 \\
15 & 0.0365 & 0.0182 & 0.0091 & 0.00068 & 0.00017 & 0.00004 \\
20 & 0.0278 & 0.0139 & 0.0069 & 0.00038 & 0.00009 & 0.00002 \\
\hline
\end{tabular}

Table 2. Numerical values of $\mathbb{E}\left[\hat{\tilde{I}}^{w}\left(F_{X_{(n: n)}}, F\right)\right]$ and $\operatorname{Var}\left[\hat{\tilde{I}}^{w}\left(F_{X_{(n: n)}}, F\right)\right]$ for beta distribution.

\begin{tabular}{|ccc|ccc|}
\hline \multicolumn{2}{|c|}{$\mathbb{E}\left[\hat{\tilde{I}}^{w}\left(F_{X_{(n: n)}}, F\right)\right]$} & \multicolumn{3}{|c|}{$\operatorname{Var}\left[\hat{\tilde{I}}^{w}\left(F_{X_{(n: n)}}, F\right)\right]$} \\
\hline $\mathrm{n}=10$ & $\mathrm{n}=15$ & $\mathrm{n}=20$ & $\mathrm{n}=10$ & $\mathrm{n}=15$ & $\mathrm{n}=20$ \\
\hline 0.00339 & 0.00166 & 0.00098 & $2.57 \mathrm{e}-15$ & $1.43 \mathrm{e}-23$ & $2.11 \mathrm{e}-32$ \\
\hline
\end{tabular}

Theorem 33

Let $\mathrm{X}$ be an absolutely continuous non-negative random variable whit $\tilde{I}^{w}\left(F_{X_{(n: n)}}, F\right)<\infty$, for all $n \geq 1$. Then we have

$$
\hat{\tilde{I}}^{w}\left(F_{X_{(n: n)}}, F\right) \longrightarrow \tilde{I}^{w}\left(F_{X_{(n: n)}}, F\right) \quad \text { a.s. }
$$

Proof

From (50) we have

$$
\begin{aligned}
\hat{\tilde{I}}^{w}\left(F_{X_{(n: n)}}, F\right) & =\int_{0}^{\infty} x\left(-\log \hat{F}_{n}(x)\right)\left(\hat{F}_{n}(x)\right)^{n} d x \\
& =\int_{0}^{1} x\left(-\log \hat{F}_{n}(x)\right)\left(\hat{F}_{n}(x)\right)^{n} d x+\int_{1}^{\infty} x\left(-\log \hat{F}_{n}(x)\right)\left(\hat{F}_{n}(x)\right)^{n} d x \\
& =: \quad R_{1}+R_{2},
\end{aligned}
$$

where

$$
\begin{aligned}
& R_{1}=\int_{0}^{1} x\left(-\log \hat{F}_{n}(x)\right)\left(\hat{F}_{n}(x)\right)^{n} d x, \\
& R_{2}=\int_{1}^{\infty} x\left(-\log \hat{F}_{n}(x)\right)\left(\hat{F}_{n}(x)\right)^{n} d x .
\end{aligned}
$$

Using dominated convergence theorem (DCT) and Glivenko-Cantelli, we have

$$
\int_{0}^{1} x\left(-\log \hat{F}_{n}(x)\right)\left(\hat{F}_{n}(x)\right)^{n} d x \longrightarrow \int_{0}^{1} x(-\log F(x))(F(x))^{n} d x \quad \text { as } m \rightarrow \infty .
$$

It follows that

$$
x^{p} \hat{F}_{n}(x) \leq \frac{1}{n} \sum_{i=1}^{n} X_{i}^{p}
$$


Morever, by using SLLN, $\quad \frac{1}{n} \sum_{i=1}^{n} X_{i}^{p} \longrightarrow \mathbb{E}\left(X^{p}\right) \quad$ and $\quad \sup _{n}\left(\frac{1}{n} \sum_{i=1}^{n} X_{i}^{p}\right)<\infty, \quad$ then $\quad \hat{F}_{n}(x) \leq$ $x^{-p}\left(\sup _{n}\left(\frac{1}{n} \sum_{i=1}^{n} X_{i}^{p}\right)\right)=C x^{-p}$. Now applying the DCT we have

$$
\lim _{n \rightarrow \infty} R_{2}=\int_{1}^{\infty} x(-\log F(x))(F(x))^{n} d x .
$$

Finally by using (55) the result follows.

In the following example, we calculate $\tilde{I}^{w}\left(F_{X_{(n: n)}}, F\right)$ and $I^{w}\left(\bar{F}_{X_{(1: n)}}, \bar{F}\right)$ for some specific lifetime distributions which are widely used in reliability theory and life testing.

\section{Example 3}

(a) If $X$ is uniformly distributed in $[0, \theta]$, then it is easy to see that $\tilde{I}^{w}\left(F_{X_{(n: n)}}, F\right)=\frac{\theta^{2}}{(n+2)^{2}}$, and $I^{w}\left(\bar{F}_{X_{(1: n)}}, \bar{F}\right)=$ $\frac{(2 n+3) \theta^{2}}{\left(n^{2}+3 n+2\right)^{2}}$, for all integers $n \geq 1$. Note that $\tilde{I}^{w}\left(F_{X_{(n: n)}}, F\right)$ and $I^{w}\left(\bar{F}_{X_{(1: n)}}, \bar{F}\right)$ are decreasing functions of $n$.

(b) If $X$ has a Weibull distribution with survival function $\bar{F}(x)=e^{-\lambda^{q} x^{q}}, x>0, \lambda, q>0$, then for all integers $n \geq 1$, we obtain $I^{w}\left(\bar{F}_{X_{(1: n)}}, \bar{F}\right)=\frac{2}{(\lambda q)^{2} n^{\frac{q+2}{q}}} \Gamma\left(\frac{2}{q}\right)$.

(c) If $X$ has a Pareto distribution with pdf $f(x)=\frac{\alpha \beta^{\alpha}}{x^{\alpha+1}}, x \geq \beta, \beta>0, \alpha>0$, then $I^{w}\left(\bar{F}_{X_{(1: n)}}, \bar{F}\right)=\frac{\alpha \beta^{2}}{(n \alpha-2)^{2}}$, for all integers $n>\frac{2}{\alpha}$. Note that $I^{w}\left(\bar{F}_{X_{(1: n)}}, \bar{F}\right)$ is a decreasing function of $n$ for all $\alpha>\frac{2}{n}$.

(d) Let $X$ be an exponential distribution with mean $\frac{1}{\lambda}$, then $I^{w}\left(\bar{F}_{X_{(1: n)}}, \bar{F}\right)=\frac{2}{n^{3} \lambda^{2}}$. Note that $I^{w}\left(\bar{F}_{X_{(1: n)}}, \bar{F}\right)$ is a decreasing function of $n$.

(e) Let $X$ be a non-negative random variable which has an inverse Weibull distribution with the cdf $F(x)=$ $\exp \left(-\left(\frac{\alpha}{x}\right)^{\beta}\right), x>0$, then for all integers $n \geq 1$, we obtain $\tilde{I}^{w}\left(F_{X_{(n: n)}}, F\right)=\frac{\alpha^{2} n^{\frac{2-\beta}{\beta}}}{\beta} \Gamma\left(\frac{\beta-2}{\beta}\right)$.

\section{Conclusion}

In this paper, we discussed on concept of a weighted cumulative residual inaccuracy measure between $\bar{F}_{X(1: n)}$ and $\bar{F}$ and studied some properties of its. We proposed a dynamic version of WCRI and studied characterization results of it. It is also proved that $I^{w}\left(\bar{F}_{X_{(1: n)}}, \bar{F} ; t\right)$ can uniquely determine the parent distribution $F$. Moreover, we studied some new basic properties of $I^{w}\left(\bar{F}_{X_{(1: n)}}, \bar{F}\right)$ such as the effect of linear transformation, relationships with other reliability functions, bounds and stochastic order properties. We estimated the WCRI by means of the empirical cumulative inaccuracy for minimum of order statistics and proved that $\hat{I}^{w}\left(\bar{F}_{X_{(1: n)}}, \bar{F}\right)$ converge to $I^{w}\left(\bar{F}_{X_{(1: n)}}, \bar{F}\right)$. Finally, similarly, we proposed the WCPI measure between $F_{X(n: n)}$ and $F$. We also studied some properties of $\tilde{I}^{w}\left(F_{X_{(n: n)}}, F\right)$ such as the connections with other reliability functions, several useful bounds and stochastic orderings. These concepts can be applied in measuring the weighted inaccuracy contained in the associated residual (past) lifetime.

\section{Acknowledgement}

The author would like to thank the Associate Editor, and anonymous referees for their valuable comments, and suggestions on improving this article.

\section{REFERENCES}

1. B.C. Arnold, N. Balakrishnan, and H. N. Nagaraja, A first course in order statistics, John Wiley, New York, 1992.

2. S. Baratpour, Characterizations based on cumulative residual entropy of first-order statistics, Communications in Statistics-Theory and Methods, vol. 39, no. 20, pp. 3645-3651, 2010.

3. C. Cali, M. Longobardi, and J. Navarro, Properties for generalized cumulative past measures of information, Probability in the Engineering and Informational Sciences, pp. 1-20, 2018. 
4. S. Danshi, A.Nezakati, and S, Tahmasebi, On weighted cumulative past (residual) inaccuracy for record values, Journal of Inequalities and Applications, vol. 2019, no. 1, pp.134, 2019.

5. M. Eskandarzadeh, A. Di Crescenzo, and S, Tahmasebi, Cumulative measure of inaccuracy and mutual information in k-th lower record values, Mathematics, vol. 7, no. 2, pp.175, https://doi.org/10.3390/math7020175, 2019.

6. A. Di Crescenzo, and M. Longobardi, On weighted residual and past entropies, Scientiae Mathematicae Japonicae, vol. 64, no. 2, pp. 255-266, 2006.

7. A. Di Crescenzo, and M. Longobardi, On cumulative entropies, Journal of Statistical Planning and Inference, vol. 139, pp. 40724087, 2009.

8. R. C. Gupta, and S. N. U. A. Kirmani, Characterization based on convex conditional mean function, Journal of Statistical Planning and Inference, vol. 138, no. 4, pp. 964-970, 2008

9. S. Kayal, On weighted generalized cumulative residual entropy of order n, Methodology and Computing in Applied Probability, DOI 10.1007/s11009-017-9569-0, pp. 1-17, 2017.

10. S. Kayal, and R. Moharana, On weighted measures of cumulative entropy, International Journal of Mathematics and Statistics, vol 18 , no. 3, pp. 26-46, 2017

11. V. Kumar, and H. C. Taneja, Dynamic cumulative residual and past inaccuracy measures, J. Stat. Theory Appl, vol. 14, no. 4, pp. 399-412, 2015.

12. F. Misagh, and G.H. Yari, On weighted interval entropy, Statistics and probability letters, vol. 81, no.2, pp. 188-194, 2011.

13. J. Navarro, Y. del Aguila and M. Asadi, Some new results on the cumulative residual entropy, Journal of Statistical Planning and Inference, vol. 140, pp. 310-322, 2010.

14. R. Pyke, Spacings, Journal of Royal Statistical Society, Series B, vol. 27, no. 3, pp. 395-449, 1965.

15. M. Rao, More on a new concept of entropy and information, Journal of Theoretical Probability, vol. 18, pp. 967-981, 2005.

16. M. Rao, Y. Chen, B. C. Vemuri, and F. Wang, Cumulative Residual Entropy: A New Measure of Information, IEEE Trans Inf Theory, vol. 50, no. 6, pp. 1220-1228, 2004.

17. M. Shaked, and J. G. Shanthikumar, Stochastic orders, Springer Science and Business Media, 2007.

18. C. E. Shannon, A mathematical theory of communication, Bell Syst. Tec. J, vol. 27, no. 3, pp. 379-432, 1948.

19. S. Tahmasebi, and S. Daneshi, Measures of inaccuracy in record values, Commun. Stat., Theory Methods, vol. 47, no. 24, pp. 6002-6018, 2018.

20. S. Tahmasebi, A.Nezakati, and S. Daneshi, Results on cumulative measure of inaccuracy in record values, J. Stat. Theory Appl, vol. 17 , no. 1 , pp. $15-28,2018$

21. R. Thapliyal, and H. C. Taneja, On residual inaccuracy of order statistics, Statist. Probab. Lett, vol. 97, pp. 125-131, 2015.

22. V, Zardasht, Results on relative mean residual life and relative cumulative residual entropy, Statistics, Optimization and Information Computing, vol. 7, no. 1, pp. 150-159, 2019. 